\section{Decontamination of Extracorporeal Membrane Oxygenator Devices With an Intensified Disinfection Protocol: How Strict Is Too Strict?}

To the Editor-In a recently published article, Garvey et $\mathrm{al}^{1}$ describe the successful water decontamination from mycobacteria in an extracorporeal membrane oxygenator device (ECMO) when applying an intensified weekly disinfection protocol.

The study refers to the intensified disinfection protocol for the Maquet ECMO Heater Unit 35 device (HU35, Rastatt, Germany), released in September 2016. ${ }^{2}$ This new maintenance guideline requires HU35s to be disinfected after each patient and at least every 7 days, compared to a monthly disinfection cycle recommended previously. Also, the intensification of the protocol depends on whether atypical mycobacteria were previously detected in a given device. ${ }^{2}$ These measures were put in place following reports of invasive and fatal infections with atypical mycobacteria transmitted by heater-cooler devices (HCDs) in cardiac surgery.,

We call the proportionality of these measures into question. First, there is currently no conclusive link between HU35 colonization and human disease. ${ }^{1,5}$ Moreover, we do not know whether the intensified protocol completely eliminates mycobacteria or just suppresses them below the detection limit. Finally, the manual states that the HU35 must be disinfected every 7 days, even if the ECMO device is in use. ${ }^{2}$ Because the oxygenator is part of the heater-cooler water circuit, it is not sufficient to disinfect only the HU35 device. Consequently, the complete ECMO system would have to be separated from the patient and exchanged after 7 days of treatment. In addition to causing substantial costs for disposable materials, this is a risky maneuver for patients who depend on ECMO-assisted oxygenation and/or circulation.

While applying the previous monthly disinfection regimen in our institution, Mycobacterium chimaera was isolated in some but not all of the HU35 water circuits. This finding may suggest an overall low mycobacterial concentration in the HU35 water. Also, total microorganism counts in the HU35 water were mostly in the range of drinking-water quality (Table 1).
Notably, the HU35 works differently than the incriminated HCDs used for cardiac surgery. The latter involve open water tanks and a fan for cooling, due to which infectious aerosols may be propelled from the device to the patient environment. ${ }^{3,4}$ HU35s, in contrast, are closed air tight and have no ventilation to the outside. It would, therefore, require a breach in the ECMO exchange membrane for a transmission to the patient to occur. In HCDs, this is an extraordinarily rare event. ${ }^{6}$ Even if it occurred, the higher pressure in the patient circuit would likely cause a blood spillover into the ECMO circuit but not the other way around. In the very unlikely case of a massive water/blood exchange, mycobacterial contamination would presumably be the least problem for the patient.

The time needed to clean a single HU35 device in our institution is approximately $2-2.5$ hours or at least 10 hours per week for our 5 devices. This total time does not include additional intermediate cleaning procedures for short-term ECMO use. Furthermore, costs for mycobacterial cultures and disposable materials are substantial.

We consulted with the Swiss Federal Office of Public Health, and they recognized that the updated measures are quite strict and not evidence based. In cases like this, a healthcare institution may therefore decide to deviate from the procedure suggested by the manufacturer. According to Swiss Medical Devices Ordinance articles 19 and 20, legal responsibility for a device is transferred from the manufacturer to the user in case of deviation from a maintenance protocol. ${ }^{7}$ However, according to Swiss law, the user is only liable if damage can causally be attributed to noncompliance with the manufacturer's protocol. The Swiss Agency for Therapeutic Products can examine the conformity of disinfection procedures of medical devices through on-site inspections. According to Swiss legislation, if an institution choses to deviate from a maintenance protocol, this has to be substantiated scientifically, documented, and kept on file to accommodate inspections.

To the best of our knowledge, we do not endanger our patients by continuing to adhere to the established monthly disinfection protocol. Therefore, the infection control unit and the department of intensive care medicine of our institution decided to assume joint responsibility for the protocol

тавLE 1. Settings and Results of the HU35 Sampling

\begin{tabular}{llccc}
\hline $\begin{array}{l}\text { HU35 } \\
\text { Serial No. }\end{array}$ & Sampling Date & $\begin{array}{c}\text { Last Disinfection } \\
\text { and Water Change }\end{array}$ & $\begin{array}{c}\text { Total Microorganisms } \\
\text { Per mL Water }^{\mathrm{a}}\end{array}$ & Mycobacteria $^{\mathrm{b}}$ \\
\hline 90004272 & 3 Aug 2017 & 14 Jul 17 & 30 & M. chimaera \\
90004206 & 3 Aug 2017 & 4 Jul 17 & 65 & No growth \\
90004385 & 3 Aug 2017 & 26 Jul 17 & 540 & M. chimaera \\
90004559 & 3 Aug 2017 & 2 Aug 17 & 5 & No growth \\
\hline
\end{tabular}

NOTE. HU35, heater unit 35 device.

${ }^{\mathrm{a}} \mathrm{A} 200 \mu \mathrm{L}$ volume was plated on blood agar supplemented with $2 \%$ sheep blood and incubated for 4 days at $36^{\circ} \mathrm{C}$.

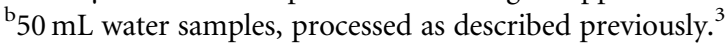


deviation. A corresponding document was created, signed, and filed by the parties involved. The document will be scrutinized as new evidence becomes available.

In conclusion, it may be possible to deviate from a disinfection protocol that is perceived as too strict, provided that the deviation is well justified and does not jeopardize patient safety. Should other institutions decide to take a similar approach, local legislation and other particularities need to be taken into consideration.

\section{ACKNOWLEDGMENTS}

We would like to thank Walter Steiger (Infection Control, Bern University Hospital) and Felix Zürcher (Intensive Care Medicine, Bern University Hospital) for technical support with HU35 sample handling.

Financial support: No financial support was provided relevant to this article.

Potential conflicts of interest: All authors report no conflicts of interest relevant to this article.

\section{Rami Sommerstein, MD; ${ }^{1}$ Tobias M. Merz, MD; Stephan M. Jakob, MD, $\mathrm{PhD}{ }^{2}$ Jukka Takala, MD, $\mathrm{PhD}{ }^{2}$ Jonas Marschall, MD, MSc}

Affiliations: 1. Department of Infectious Diseases, Bern University Hospital, University of Bern, Switzerland; 2. Department of Intensive Care Medicine, Bern University Hospital, University of Bern, Switzerland.

Address correspondence to Rami Sommerstein, MD, Department of Infectious Diseases, Bern University Hospital, University of Bern, Freiburgstrasse, 3010 Bern, Switzerland (rami.sommerstein@insel.ch).

Infect Control Hosp Epidemiol 2018;39:366-367

(c) 2018 by The Society for Healthcare Epidemiology of America. All rights reserved. 0899-823X/2018/3903-0023. DOI: 10.1017/ice.2017.283

\section{REFERENCES}

1. Garvey MI, Phillips N, Bradley CW, Holden E. Decontamination of an extracorporeal membrane oxygenator contaminated with Mycobacterium chimaera. Infect Control Hosp Epidemiol 2017;38: 1244-1246.

2. Heater Unit HU35. Revised instructions for use-cleaning, descaling, disinfection. 1.0.XX.01. Maquet Cardiopulmonary GmbH.

3. Sax H, Bloemberg G, Hasse B, et al. Prolonged outbreak of Mycobacterium chimaera infection after open-chest heart surgery. Clin Infect Dis 2015;61:67-75.

4. Sommerstein R, Ruegg C, Kohler P, Bloemberg G, Kuster SP, Sax H. Transmission of Mycobacterium chimaera from heatercooler units during cardiac surgery despite an ultraclean air ventilation system. Emerg Infect Dis 2016;22:1008-1013.

5. Trudzinski FC, Schlotthauer U, Kamp A, et al. Clinical implications of Mycobacterium chimaera detection in thermoregulatory devices used for extracorporeal membrane oxygenation (ECMO), Germany, 2015 to 2016. Euro Surveill 2016;21.

6. Mejak BL, Stammers A, Rauch E, Vang S, Viessman T. A retrospective study on perfusion incidents and safety devices. Perfusion 2000;15:51-61.

7. Medical devices ordinance. The Swiss Federal Council website. https://www.admin.ch/opc/en/classified-compilation/19995459/ index.html. Published 2001. Accessed, November 9, 2017.

\section{Staphylococcus aureus Bloodstream Infection Due to Contaminated Hematopoietic Stem-Cell Graft}

To the Editor-The Foundation for the Accreditation of Cellular Therapy and the American Association of Blood Banks publish guidelines to ensure the quality and safety of hematopoietic stem-cell (HSC) products. ${ }^{1,2}$ These HSC products are generally cultured after procurement by the collection facility and following processing at the transplant center. Reported contamination rates of HSC grafts range from $1 \%$ to $45 \% .^{3-5}$ The clinical significance of infusion of contaminated HSC products is unclear. When fresh products are used, contamination is often not identified prior to HSC infusion. Bacterial contamination is not an absolute contraindication to HSC infusion, as options are limited following a myeloablative preparative regimen. In a review of 12 studies, $91 \%$ of contaminated grafts contained bacterial species of low pathogenicity (eg, Staphylococcus epidermidis and Propionibacterium acnes). Of 26 patients who received grafts contaminated with highly pathogenic bacteria (eg, S. aureus), none developed symptoms or had a positive culture matching an organism found in the HSC graft. ${ }^{3}$ In prior reports of infections putatively caused by graft contamination, confirmation that the graft was the source of infection was based solely on the finding of identical species. ${ }^{6,7}$ Contrary to these prior reports, we present a case of catheter-related bloodstream infection with methicillinsusceptible S. aureus due to a contaminated HSC graft in which pulsed-field gel electrophoresis (PFGE) confirmed that the graft and patient isolates were identical.

A 15-year-old male presented for hematopoietic cell transplant (HCT) for hypodiploid B-cell acute lymphoblastic leukemia (B-ALL). His history included osteosarcoma of the proximal right tibia, for which he had completed treatment with chemotherapy and limb-sparing resection two years prior to this admission. At the time B-ALL was diagnosed, local recurrence of osteosarcoma was also discovered in the distal right femur. He began therapy for both cancers per the Children's Cancer Group protocol 1941 with modifications as appropriate. He underwent a right transfemoral amputation and achieved remission of osteosarcoma.

After achieving complete remission of B-ALL, he underwent a 10/10 HLA-allele matched unrelated donor HCT. Preparative regimen per Children's Oncology Group protocol AALL1331 included fractionated total body irradiation, thiotepa, and cyclophosphamide. Graft-versus-host disease prophylaxis included tacrolimus and methotrexate. Antimicrobial prophylaxis included posaconazole, valacyclovir, pentamidine, and levofloxacin. Marrow was collected at an outside collection center approximately 24 hours prior to infusion, and the product remained at room temperature. On day 0 , the patient received the fresh donor marrow via the red lumen of his tunneled double-lumen central venous catheter (DLCVC). Infusion was complicated by hypertension and bradycardia, managed with furosemide and hydralazine. 